\title{
A New Optimal Nonlinear Approach to Half Car Active Suspension Control
}

\author{
Iraj Hassanzadeh, Ghasem Alizadeh, Naser Pourqorban Shirjoposht, Farzad Hashemzadeh
}

\begin{abstract}
In this paper, a nonlinear optimal control law based on a quadratic cost function is developed, and applied on a half-car model for the control of active suspension systems. Nonlinear model of half-car is constructed using the nonlinear dynamics of the electro hydraulic actuator and dynamic characteristics of the dampings and springs. The states of half car model are first estimated by Extended Kalman Filter (EKF) and then the estimated states predicted by Taylor series expansion and finally a control law is introduced by minimizing the local differences between the predicted and desired states. The derived control law has an analytical form which is easy to apply and also it is not required online numerical computations in optimization. Performance of the nonlinear optimal controller is compared to the existing passive suspension system and the proportional integral sliding mode controller. The obtained results demonstrate that use of the proposed nonlinear optimal control technique improves the tradeoff between ride quality and suspension travel compared to the passive suspension system and the proportional integral sliding mode method.
\end{abstract}

Index Terms - nonlinear optimal control, active suspension, nonlinear model, Extended Kalman Filter, proportional integral sliding mode.

\section{INTRODUCTION}

The important functionality of the vehicle suspension system is to support the vehicle body as well as to provide the riding comfort to the passengers by rejecting the unpleasant vibratory motion induced from the irregular road inputs. Also, the suspension should maintain adequate vertical load to provide the vehicle stability when the car turns, brakes or accelerates [1]. The vehicle stability and riding comfort has mutually adverse effects, therefore, simply the passive damper, which is widely used in the usual vehicle, could not satisfy the riding comfort as well as driving stability simultaneously. To overcome this problem many researchers have proposed to use active suspensions [2]. Unlike passive systems which can only store or dissipate energy, active suspensions can continuously change the energy flow to or from the system when required. Furthermore, characteristics of active suspensions can adapt to instantaneous changes in driving conditions detected by sensors. As a result active suspensions can improve both riding comfort and handling performance to satisfactory levels.

Manuscript received January 25, 2010

Authors are with Control Engineering Department, Faculty of Electrical and computer Engineering, University of Tabriz. Tabriz, IRAN. (Phone: +98-411-339 3728; fax: +98-411-3300819; e-mail: izadeh@ tabrizu.ac.ir, izadeh@ieee.com).
However, as the recent trends of the vehicle industry is to be luxurious and driver comfort is more required, the electrical controlled suspension system is now installed and widely utilized [3]. Especially, the active damper is widely adopted for the luxurious vehicle because it can overcome the shortage of the passive damper by providing the ride comfort and vehicle stability at the same time. In the active suspension system, the sprung mass can vary according to the variation of passenger numbers and cargo as well as fuel loading condition. Also the actuator itself has the strong non-linear characteristics and the fluctuations of the hydraulic system's parameters are not small, i.e. fluid compressibility, fluid leakage and electrical servo components.

Various control strategies such as optimal control [4], nonlinear control [5], robust control [6], adaptive control [7] and intelligent control [8] have been proposed in the past years to control the active suspension system. Most control methods have been used for active suspension systems are optimal control method [9], [10]. In these optimal control methods a linear model for automotive proposed and different optimal control approaches such as linear quadratic regulator and linear quadratic gaussian have been used. But real model of automotive is nonlinear. Therefore for a good design, it is necessary that an actual model for automotive used. In this paper, according to system requirements, an optimal nonlinear approach [11-13] is applied. Similar to this method has been used for control of yaw dynamics to improve vehicle lateral stability [14], [15]. The proposed controller has two distinguished features: firstly, it is based on continuous nonlinear model and can handle the model nonlinearity successfully. Secondly, the optimality of the control law provides the possibility of using lower control energy for achievement of the desired performance. In this paper, a new optimal predictive approach is utilized to design a non-linear controller. This method, which employs a nonlinear continuous-time dynamic model, leads to an analytical closed-form control law which is suitable to implement. The rest of the paper is organized as follows:

The model of a half-car suspension system and dynamics of it is initially investigated in section two. Then in the section three, an optimization-based nonlinear control law is developed. The main properties of the proposed controller and its advantages than the other conventional control methods are discussed. Some comparative simulation results including a passive suspension and active suspension system which is based on proportional integral sliding mode control (PISMC) theory and our resulting active suspension system are 
illustrated in section four. Section five contains the conclusions of the paper.

\section{SUSPENSION MODEL}

The well-known rigid half-car vehicle model, which is shown in Figure 1, is widely used for active suspension design. The model comprises three parts: the sprung mass and two unsprung masses. Let the sprung and unsprung masses be denoted by $M, m_{f}, m_{r}$, respectively. The half-car model is a four degrees-of-freedom system. The sprung mass is assumed to be a rigid body and has freedoms of motion in the vertical and pitch direction. The $Z_{c}$ denotes the vertical displacement at the center of gravity and $\theta$ is the pitch angle of the sprung mass. The front and rear displacements of the sprung and the unsprung masses are denoted by $Z_{f}, Z_{r}$ and $Z_{1}, Z_{2}$. In the model, the disturbances, $Z_{01}, Z_{02}$ are caused by road irregularities. The control signals, $u_{f}(t), u_{r}(t)$ are generated by the actuators.

Using Newton's second law the equations of motion can are given as:

$$
\begin{aligned}
& M \ddot{z}_{c}=f_{f}+f_{r} \\
& I \ddot{\theta}=f_{f} a-f_{r} b \\
& m_{f} \ddot{z}_{1}=-k_{f 2}\left(z_{1}-z_{01}\right)-f_{f} \\
& m_{r} \ddot{z}_{2}=-k_{r 2}\left(z_{2}-z_{02}\right)-f_{r} \\
& f_{f}=F_{k_{f 1}}+F_{b_{f}}+u_{f} \\
& f_{r}=F_{k_{r 1}}+F_{b_{r}}+u_{r} \\
& F_{k_{i 1}} \text { and } F_{b_{i}}(i \in\{f, r\}) \text { are as follows: } \\
& F_{k_{f 1}}=k_{f 1}^{l}\left(z_{1}-z_{c}-a \theta\right)+k_{f 1}^{n l}\left(z_{1}-z_{c}-a \theta\right)^{3} \\
& F_{k_{r 1}}=k_{r 1}^{l}\left(z_{2}-z_{c}+b \theta\right)+k_{r 1}^{n l}\left(z_{2}-z_{c}+b \theta\right)^{3} \\
& F_{b_{f}}=b_{f}^{l}\left(\dot{z}_{1}-\dot{z}_{c}-a \dot{\theta}\right) \\
& +b_{f}^{n l}\left(\sqrt{\left(\dot{z}_{1}-\dot{z}_{c}-a \dot{\theta}\right) \mid} \operatorname{sign}\left(\dot{z}_{1}-\dot{z}_{c}-a \dot{\theta}\right)-\left|\left(\dot{z}_{1}-\dot{z}_{c}-a \dot{\theta}\right)\right|\right) \\
& F_{b_{r}}=b_{r}^{l}\left(\dot{z}_{2}-\dot{z}_{c}+b \dot{\theta}\right) \\
& +b_{r}^{n l}\left(\sqrt{\left.\left|\left(\dot{z}_{2}-\dot{z}_{c}+b \dot{\theta}\right)\right| \operatorname{sign}\left(\dot{z}_{2}-\dot{z}_{c}+b \dot{\theta}\right)-\left|\left(\dot{z}_{2}-\dot{z}_{c}+b \dot{\theta}\right)\right|\right)}\right.
\end{aligned}
$$

In these equations $k_{f 1}, k_{r 1}$ and $k_{f 2}, k_{r 2}$ are front and rear nonlinear suspension stiffness and the front and rear linear tire stiffness, respectively. The front and rear nonlinear suspension dampings are denoted by $b_{f}, b_{r}$. The suspension spring forces at the front and rear and suspension damping forces at front and rear are denoted by $F_{k_{f 1}}, F_{k_{r 1}}$ and $F_{b_{f}}$, $F_{b_{r}}$ respectively. The nonlinear nonlinear suspension stiffness $k_{i 1}(i \in\{f, r\})$ are combination of a linear coefficient $k_{i 1}^{l}$ and a nonlinear $k_{i 1}^{n l}$ and also the nonlinear suspension damping $b_{i}(i \in\{f, r\})$ are made of $b_{i}^{l}$ and $b_{i}^{n l}$.

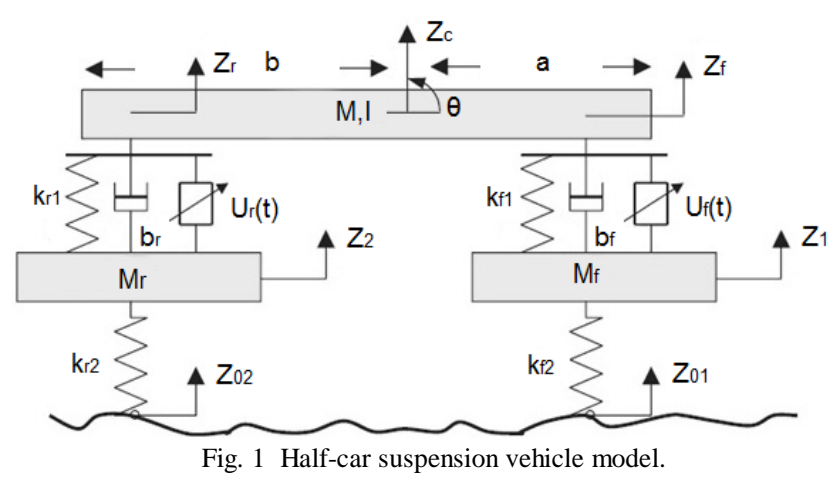

Hydraulic actuators are used for suspension systems to generate the pushing force between the sprung and unsprung masses. Let the hydraulic actuator used for suspension model be a four-way valve-piston system, in which the force $u_{i}$ is $u_{i}(t)=A P_{L i}$

where $A$ is the area of piston and $P_{L i}$ is the pressure drop across the piston with respect to the front and rear suspensions ( $i \in\{f, r\}$ ) [16]. The derivative of $P_{L i}$ is given by Eqs. (7) and (8).

$$
\begin{aligned}
& \frac{V_{t}}{4 \beta_{e}} \dot{P}_{L f}=Q_{f}-C_{t p} P_{L f}-A\left(\dot{z}_{c}+a \dot{\theta}-\dot{z}_{1}\right) \\
& \frac{V_{t}}{4 \beta_{e}} \dot{P}_{L r}=Q_{r}-C_{t p} P_{L r}-A\left(\dot{z}_{c}-b \dot{\theta}-\dot{z}_{2}\right)
\end{aligned}
$$

where $V_{t}$ is the total actuator volume, $\beta_{e}$ is the effective bulk modulus of system, $Q_{i}(i \in\{f, r\})$ is the hydraulic load flow, and $C_{t p}$ is the total leakage coefficient of piston.

The relationship between the spool valve displacement and the hydraulic load flow is given as:

$Q_{i}=\operatorname{sign}\left[P_{s}-\operatorname{sign}\left(x_{v i}\right) P_{L i}\right] C_{d} \omega x_{v i} \sqrt{\frac{1}{\bar{\rho}}\left|P_{s}-\operatorname{sign}\left(x_{v i}\right) P_{L i}\right|}$

where $C_{d}$ is the discharge coefficient, $\omega$ is the spool valve area gradient, $x_{v i}$ is the displacement of spool valve, $\bar{\rho}$ is the hydraulic fluid density, and $P_{s}$ is the supply pressure. The $x_{v i}$ is controlled by the input to the servo-valve $v_{i}$. The valve dynamics are approximated by a linear filter with time constant $\tau$ :

$$
\dot{x}_{v i}=\frac{1}{\tau}\left(-x_{v i}+v_{i}\right)
$$

The nonlinear model of the hydraulic actuator is as follows:

$$
\begin{aligned}
& \dot{P}_{L f}=\alpha Q_{f}-\beta P_{L f}-\alpha A\left(\dot{z}_{c}+a \dot{\theta}-\dot{z}_{1}\right) \\
& \dot{P}_{L r}=\alpha Q_{r}-\beta P_{L r}-\alpha A\left(\dot{z}_{c}-b \dot{\theta}-\dot{z}_{2}\right) \\
& \alpha Q_{i}=\gamma \operatorname{sign}\left[P_{s}-\operatorname{sign}\left(x_{v i}\right) P_{L i}\right] x_{v i} \sqrt{\left|P_{s}-\operatorname{sign}\left(x_{v i}\right) P_{L i}\right|} \\
& \dot{x}_{v i}=\frac{1}{\tau}\left(-x_{v i}+v_{i}\right)
\end{aligned}
$$

where $\alpha=\frac{4 \beta_{e}}{V_{t}}, \beta=\alpha C_{t p}, \gamma=\frac{C_{d} \omega}{\sqrt{\bar{\rho}}} \alpha$

If defined the state variable, $X$, the control input, $V$, the disturbance input, $W$ as: 
$x_{1}=z_{c}+a \theta-z_{1} \quad x_{2}=\dot{z}_{c}+a \dot{\theta} \quad x_{3}=z_{c}-b \theta-z_{2}$

$x_{4}=\dot{z}_{c}-b \dot{\theta} \quad x_{5}=z_{1}-z_{01} \quad x_{6}=\dot{z}_{1} \quad x_{7}=z_{2}-z_{02}$

$x_{8}=\dot{z}_{2} \quad x_{9}=\mu P_{L f} \quad x_{10}=x_{v f} \quad x_{11}=\mu P_{L r} \quad x_{12}=x_{v r}$

$V=\left[v_{1}, v_{2}\right]^{T}$

$W=\left[w_{1}, w_{2}\right]^{T}$

$\mu=10^{-6}$ is used to rescale the pressure drop, $P_{L i}$ ( $i \in\{f, r\}$ ), to improve numerical accuracy. The components of the input disturbance (the rate of change in the height of road irregularities) for front and rear roads are $w_{1}(t)=\dot{Z}_{01}(t)$ and $w_{2}(t)=w_{1}\left(t-t_{d}\right)$, where $t_{d}$ represents the time delay between front and rear tire axis. When nonlinear equations and nonlinear model of the hydraulic actuator use, the nonlinear suspension system has the following from:

$$
\dot{X}=f+B V+D W
$$

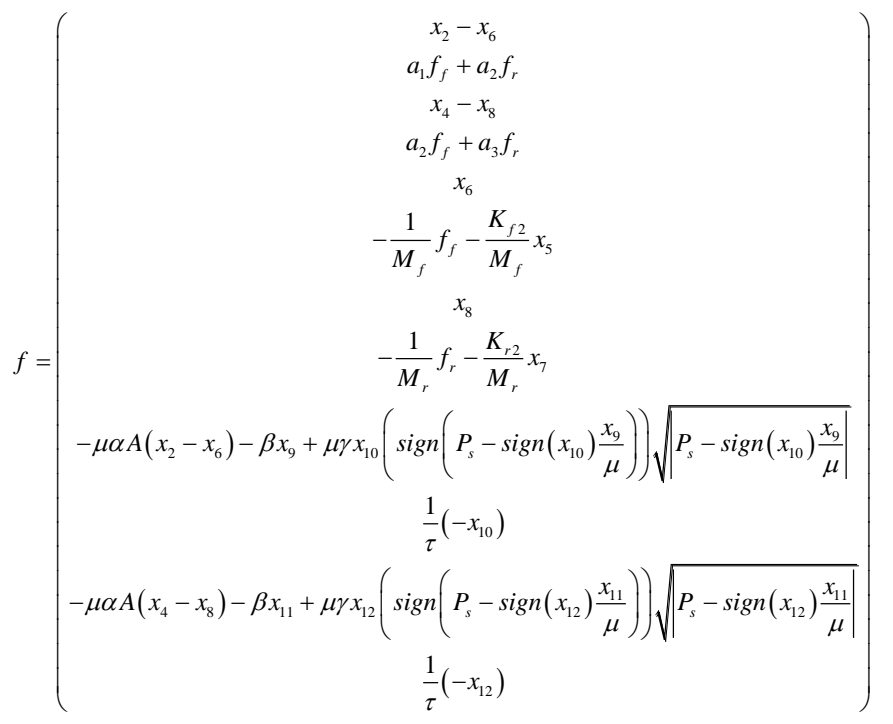

where

$f_{f}=-k_{f 1}^{l} x_{1}-k_{f 1}^{n l} x_{1}^{3}+b_{f}^{l}\left(x_{6}-x_{2}\right)+b_{f}^{n l}\left(\sqrt{\left(x_{6}-x_{2}\right) \mid} \operatorname{sign}\left(x_{6}-x_{2}\right)-\left|\left(x_{6}-x_{2}\right)\right|\right)$ $+\frac{A}{\mu} x_{9}$

$f_{r}=-k_{r 1}^{l} x_{3}-k_{r 1}^{n l} x_{3}^{3}-b_{r}^{l}\left(x_{8}-x_{4}\right)+b_{r}^{n l}\left(\sqrt{\left(x_{8}-x_{4}\right) \mid} \operatorname{sign}\left(x_{8}-x_{4}\right)-\left|\left(x_{8}-x_{4}\right)\right|\right)$ $+\frac{A}{\mu} x_{11}$

$$
\begin{aligned}
& a_{1}=\frac{1}{M}+\frac{a^{2}}{I}, a_{2}=\frac{1}{M}-\frac{a b}{I}, a_{3}=\frac{1}{M}+\frac{b^{2}}{I} \\
& B=\left[\begin{array}{llllllllllll}
0 & 0 & 0 & 0 & 0 & 0 & 0 & 0 & 0 & \frac{1}{\tau} & 0 & 0 \\
0 & 0 & 0 & 0 & 0 & 0 & 0 & 0 & 0 & 0 & 0 & \frac{1}{\tau}
\end{array}\right]^{T} \\
& L=\left[\begin{array}{cccccccccccc}
0 & 0 & 0 & 0 & -1 & 0 & 0 & 0 & 0 & 0 & 0 & 0 \\
0 & 0 & 0 & 0 & 0 & 0 & -1 & 0 & 0 & 0 & 0 & 0
\end{array}\right]^{T}
\end{aligned}
$$

\section{Control System Design}

In this section firstly the states of the system estimated by the Extended Kalman Filter (EKF). The Kalman Filter (KF) is one of the most widely used methods for tracking and estimation due to its simplicity, optimality, tractability and robustness [17]. However, the application of the KF to nonlinear systems can be difficult. The most common approach is to use the EKF which simply linearizes all nonlinear models so that the traditional linear Kalman Filter can be applied [18]. The EKF is a recursive predictive filter that is based on the use of state space techniques and recursive algorithms. This dynamic system can be disturbed by some noise, mostly assumed as white noise. To improve the estimated state the EKF uses measurements that are related to the state disturbed as well [19]. Thus the EKF consist of two steps:

1) The prediction

2) The correction

In the first step the state is predicted with the dynamic model. In the second step it is corrected with the observation model, so that the error covariance of the estimator is minimized. In this sense it is an optimal estimator. The dynamic model represented by

$$
\dot{X}(t)=f(X(t), m(t))
$$

where $m(t)$ is the noise vector. In the linear case this can easily be rewritten as

$$
\dot{X}(t)=F \cdot X(t)+n(t)
$$

where $F$ is the dynamic matrix and is constant, $X(t)$ is the state vector and $n(t)$ is the dynamic noise which is usually assumed as white noise and has the covariance matrix $Q(t)$. The observation model represents the relationship between the state and the measurements. Usually the observations are made at discrete time steps $t_{i}$

$$
l\left(t_{i}\right)=h\left(X\left(t_{i}\right), v\left(t_{i}\right)\right)
$$

where $v\left(t_{i}\right)$ is the noise of the measurement process at the epoch $t_{i}$. In the linear case the measurements can be described by a system of linear equations, which depend on the state variables. The vector from of this system is

$$
l\left(t_{i}\right)=H . X\left(t_{i}\right)+w\left(t_{i}\right)
$$

where $l\left(t_{i}\right)$ is the vector of the observations at the epoch $t_{i}$, $H$ is the observation matrix and $w\left(t_{i}\right)$ is the noise of the measurement process with the covariance matrix $R\left(t_{i}\right)$. Like the dynamic matrix, in a linear system the observation matrix $H$ is a constant matrix as well.

Like mentioned before, the prediction is the first step of the EKF. The predicted state, or better the a priori state is calculated by neglecting the dynamic noise and solving the differential equation the describe the dynamic model

$$
\dot{X}^{-}\left(t_{i}\right)=f\left(X^{-}\left(t_{i}\right)\right)
$$

By representing this equation by a Taylor series with respect to $X$ at the predicted state $X^{-}\left(t_{i}\right)$ and assuming that the higher order terms can be neglected, Eq. (20) can be rewritten as

$$
\dot{X}^{-}\left(t_{i}\right)=F\left(t_{i}\right) \cdot X^{-}\left(t_{i}\right)
$$

where

$$
F\left(t_{i}\right)=\left.\frac{\partial f(X)}{\partial X}\right|_{X=X^{-}\left(t_{i}\right)}
$$


The state vector at time $t_{i}$ can be expressed by Taylor series with respect to an approximate state $X^{-}\left(t_{0}\right)$.

$$
X^{-}\left(t_{i}\right)=X^{-}\left(t_{0}\right)+\dot{X}^{-}\left(t_{0}\right)\left(t_{i}-t_{0}\right)+\frac{1}{2} \ddot{X}^{-}\left(t_{0}\right)\left(t_{i}-t_{0}\right)^{2}+\ldots
$$

by using Eq. (21) this can be rewritten as

$$
X^{-}\left(t_{i}\right)=X^{-}\left(t_{0}\right)+F\left(t_{i}\right) X^{-}\left(t_{0}\right)\left(t_{i}-t_{0}\right)+\frac{1}{2} F^{2}\left(t_{i}\right) X^{-}\left(t_{0}\right)\left(t_{i}-t_{0}\right)^{2}+\ldots
$$

Thus the solution $X^{-}\left(t_{i}\right)$ of the differential equations, in other words the actual predicted state is a linear combination of the initial state $X^{-}\left(t_{0}\right)$

$$
X^{-}\left(t_{i}\right)=\phi_{0}^{t_{i}} \cdot X^{-}\left(t_{0}\right)
$$

$\phi_{0}^{t_{i}}$ is called the state transition matrix, which transforms any initial state $X\left(t_{0}\right)$ to its corresponding state $X\left(t_{i}\right)$ at time $t_{i}$

from the Eqs. (21) and (25)

$$
\dot{X}^{-}\left(t_{i}\right)=F\left(t_{i}\right) \cdot X^{-}\left(t_{i}\right)=F\left(t_{i}\right) \cdot \phi_{0}^{t_{i}} \cdot X^{-}\left(t_{0}\right)
$$

and by using (26) again, one can see

$$
\dot{X}^{-}\left(t_{i}\right)=\frac{d}{d t} X^{-}\left(t_{i}\right)=\frac{d}{d t}\left(\phi_{0}^{t_{i}} \cdot X^{-}\left(t_{0}\right)\right)=\frac{d}{d t}\left(\phi_{0}^{t_{i}}\right) \cdot X^{-}\left(t_{0}\right)
$$

by comparing (26) and (27) it follows that:

$$
\frac{d}{d t}\left(\phi_{0}^{t_{i}}\right)=F\left(t_{i}\right) \cdot \phi_{0}^{t_{i}}
$$

with the initial matrix $\phi_{0}^{0}=I$, because $X\left(t_{0}\right)=I . X\left(t_{0}\right)$. And now the covariance matrix $P^{-}\left(t_{i}\right)$ of the predicted state vector is obtained with the law of error propagation

$$
P^{-}\left(t_{i}\right)=\phi_{t_{i-1}}^{t_{i}} \cdot P\left(t_{i-1}\right) \cdot\left(\phi_{t_{i-1}}^{t_{i}}\right)^{T}+Q
$$

In the more generalized form, where also the covariance matrix of the noise $Q$ is a function of time, the covariance matrix is:

$$
P^{-}\left(t_{i}\right)=\phi_{t_{i-1}}^{t_{i}} \cdot P\left(t_{i-1}\right) \cdot\left(\phi_{t_{i-1}}^{t_{i}}\right)^{T}+\int_{t_{i-1}}^{t_{i}} Q(t) d t
$$

In the correction step the predicted state vector $X^{-}\left(t_{i}\right)$ is improved with observations made at the epoch $t_{i}$, thus the a posteriori state has the form

$$
X^{+}\left(t_{i}\right)=X^{-}\left(t_{i}\right)+\Delta X\left(t_{i}\right)
$$

with the covariance matrix

$$
P^{+}\left(t_{i}\right)=P^{-}\left(t_{i}\right)+\Delta P\left(t_{i}\right)
$$

As said before the EKF is an optimal filter, this means that the state variances in the state covariance matrix $P^{+}$are minimizd. As $P^{-}$is already known from the prediction step it follows that $\Delta P$ is minimized.

$$
\Delta P\left(t_{i}\right)=E\left[\Delta X\left(t_{i}\right) \Delta X\left(t_{i}\right)^{T}\right]
$$

This condition is complied with

$$
\begin{aligned}
& \Delta X\left(t_{i}\right)=P^{-}\left(t_{i}\right) H^{T}\left(t_{i}\right)\left(H\left(t_{i}\right) P^{-}\left(t_{i}\right) H^{T}\left(t_{i}\right)+R\left(t_{i}\right)\right)^{-1} \cdot\left(l\left(t_{i}\right)-H\left(t_{i}\right) X^{-}\left(t_{i}\right)\right)(34) \\
& \Delta X\left(t_{i}\right)=K\left(t_{i}\right) \cdot\left(l\left(t_{i}\right)-H\left(t_{i}\right) X^{-}\left(t_{i}\right)\right)
\end{aligned}
$$

with

$$
K\left(t_{i}\right)=P^{-}\left(t_{i}\right) H^{T}\left(t_{i}\right)\left(H\left(t_{i}\right) P^{-}\left(t_{i}\right) H^{T}\left(t_{i}\right)+R\left(t_{i}\right)\right)^{-1}
$$

$K\left(t_{i}\right)$ is called the gain matrix. The difference $\left(l\left(t_{i}\right)-l^{-}\left(t_{i}\right)\right)$ is called the measurement residual. It reflects the discrepancy between the predicted measurement $l^{-}\left(t_{i}\right)=H X^{-}\left(t_{i}\right)$ and the actual measurement $l\left(t_{i}\right)$.

Finally the corrected state is obtained by

$X^{+}\left(t_{i}\right)=X^{-}\left(t_{i}\right)+K\left(t_{i}\right) \cdot\left(l\left(t_{i}\right)-l^{-}\left(t_{i}\right)\right)$

In this equation the estimated state and the measurements are weighted and combined to calculate the corrected state. That means, if the measurement covariance is much smaller than that of the predicted state, the measurements weight will be high and the predicted states will be low. And so the uncertainty can be reduced. The covariance matrix of the a posteriori state is given with the law of error propagation by $P^{+}\left(t_{i}\right)=P^{-}\left(t_{i}\right)-K\left(t_{i}\right) H\left(t_{i}\right) P^{-}\left(t_{i}\right)=\left(I-K\left(t_{i}\right) H\left(t_{i}\right)\right) P^{-}\left(t_{i}\right)$

In this paper distance of front and rear of automobile to surface of road are considered as a measurable output for numerical calculation. Now, we are ready for to design of the controller. The main goal of the control system is to make the estimated states $x_{n}(n=1,2, \ldots, 12)$ to follow the desired state $x_{n d}$. Briefly, the estimated states for the next time interval, $x_{n}(t+h)$, is first predicted by Taylor series expansion and then the current control $v_{m}(m=1,2)$ will be found based on continuous minimization of predicted tracking error. Note that $h$ denotes to the predictive period and is a real positive number. Let us first approximate $x_{n}(t+h)$ by a $k$ th-order Taylor series at $t$ :

$$
\begin{aligned}
& x_{n}(t+h)=x_{n}(t)+h \dot{x}_{n}(t)+\frac{h^{2}}{2 !} \ddot{x}_{n}(t)+\ldots+\frac{h^{k}}{k !} x_{n}{ }^{(k)}(t) \\
& x_{n}(t+h)=\zeta\left(X(t), \dot{V}(t), \ldots, V^{k-1}(t), \dot{W}(t), \ldots, W^{k-1}(t)\right)
\end{aligned}
$$

Now, the key issue is to choose the order $k$ in a way which is suitable for the purposes of controller design on the basis of predictions. The expansion order $k$ is determined as the lowest order of the derivative of state $x_{n}$ in which the input $V$ first appears explicitly. Hence, state vector $X(t+h)$ is as follows:

$$
X(t+h)=L_{1}\left(t, X(t), W(t), \dot{W}(t), \ldots, W^{k-1}(t)\right)+L_{2}(t, X(t)) V(t)
$$

Note that the arguments of functions may be frequently dropped through the rest of paper for simplicity of notations. Now, we consider a performance index that penalizes the next instant tracking error and the current control expenditure in the following form:

$$
J[V(t)]=\frac{1}{2}\left(X(t+h)-X_{d}(t+h)\right)^{T} W_{1}\left(X(t+h)-X_{d}(t+h)\right)+\frac{1}{2} V(t)^{T} W_{2} V(t)(40)
$$

where $W_{1}$ and $W_{2}$ are symmetric positive semi-definite and symmetric positive definite weighting matrices, respectively. Minimization of the performance index must be sought in order to improve the tracking accuracy of states at the next instant and consequently obtain the optimum behavior of the vehicle. Now, the expanded performance index can be obtained as a function of control input by substituting Eq. (39) into (40) as:

$$
J[V(t)]=\frac{1}{2}\left(L_{1}+L_{2} V-X_{d}\right)^{T} W_{1}\left(L_{1}+L_{2} V-X_{d}\right)+\frac{1}{2} V(t)^{T} W_{2} V(t)
$$

The necessary condition for optimality is 


$$
\frac{\partial J}{\partial V}=0=L_{2}^{T} W_{1}\left(L_{1}+L_{2} V-X_{d}\right)+W_{2} V
$$

which, leads to

$$
V=-\left(L_{2}^{T} W_{1} L_{2}+W_{2}\right)^{-1} L_{2}^{T} W_{1}\left(L_{1}-X_{d}\right)
$$

It is considered that the analytically defined predictive control law, Eq. (43), is a closed form which depends on the states of the estimated system and disturbance input. Generally, the proposed control law has two free parameters: the predictive time $h$ and the weighting matrices $W_{1}$ and $W_{2}$. The dynamic performance of the controller is extremely sensitive to the values of these parameters. In the derived control law, the predictive period $h$ is treated as a controller parameter rather than the integration step size. This feature is not shared by discrete-time systems [20]. Also, it can be established that a certain degree of robustness in the presence of some modeling uncertainties is achievable through small values of $h$. We see that the proposed tracking controller technique naturally leads to a special case of feedback linearization. But the current control law (43) has some important advantages over the input/output linearization control. It can be established that the predictive controller is robust in the presence of a class of modeling uncertainties and doesn't need the exact knowledge of the system nonlinearity unlike the feedback linearization. Optimal property of the proposed control law is another important advantage that provides the possibility of limiting the control by regulation of weighting matrices.

\section{Simulation AND DISCUSSION}

Computer simulations are carried out to verify the effectiveness of the designed nonlinear optimal control system. The vehicle parameters for a compact sedan that are used in simulation are listed in Table 1 . Matrices $W_{1}, W_{2}$ and the predictive period $h$ of the Nonlinear Optimal control and other parameters are accurately regulate for the computer simulations. Let the set of typical road disturbance be in the form of

$$
Z_{01}(t)=\left\{\begin{array}{cc}
a(1-\cos (8 \pi t)) & 0.5 \leq t \leq 0.75 \\
b(1-\cos (8 \pi t)) & 3 \leq t \leq 3.25 \\
0 & \text { other wise }
\end{array}\right.
$$

where $a$ and $b$ denote the bump amplitude (Fig. 2). This type of road disturbance has been used by [21], [22] in their studies. Furthermore, the maximum travel distance of the suspension travel is $\pm 8 \mathrm{~cm}$ as suggested in [22]. For comparison purposes, the performance of the designed nonlinear optimal control is compared to the proportional integral sliding mode control (PISMC) approach. For the system in Eq. (15), we utilized the PI sliding surface defined as

$$
\text { follows: } \sigma(t)=C X(t)+\int_{0}^{t}(C E) X(\eta) d \eta
$$

where $C$ (dimension [ $C]=m \times n)$ and $E$ (dimension $[E]=n \times n)$ are constant matrices. It is well known that if the system is able to enter the sliding mode, hence $\sigma(t)=0$. Therefore the equivalent control, $V_{e q}(t)$ can thus be obtained by letting $\dot{\sigma}(t)=0$ [23].

Table 1: Parameters of half car model

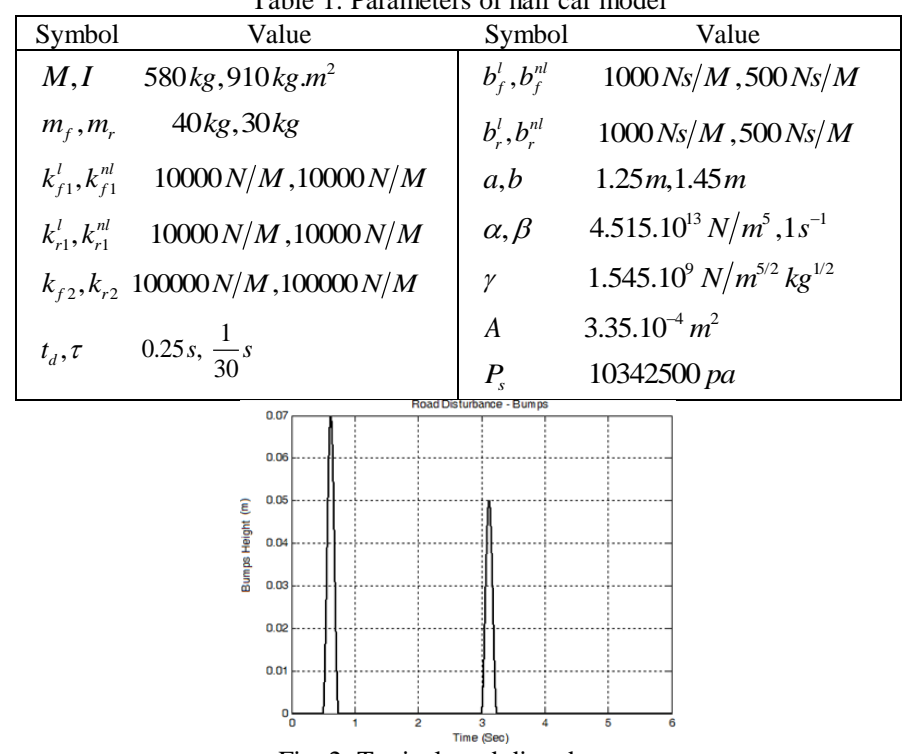

Fig. 2 Typical road disturbance.

We design the control scheme that drives the state trajectories of the system in Eq. (15) onto the sliding surface $\sigma(t)=0$ and the system remains in it thereafter. For the system in Eq. (15), the following control law is proposed:

$$
\begin{aligned}
& V(t)=-(C B)^{-1} C(f+D W)-(C B)^{-1}(C E) X(t) \\
& -(C B)^{-1} \phi \sigma(t)-(C B)^{-1} \varepsilon \cdot \operatorname{sign}(\sigma(t))
\end{aligned}
$$

where $\phi \in \mathfrak{R}^{m \times m}$ is a positive symmetric design matrix and $\varepsilon$ is the positive constant.

In order to fulfill the objective of designing an active suspension system, i.e., to increase the ride comfort and road handling, there are two parameters to be observed in the simulations. The two parameters are the car body acceleration and the wheel deflection.

Fig. 3 shows the suspension travels of both controllers for an active suspension system and a passive suspension system for comparison purposes. The result shows that the suspension travel within the travel limit, i.e., $\pm 8 \mathrm{~cm}$ and the result also shows that the active suspension utilizing the proposed controller, performs better as compared to the others.

Fig. 4 illustrates clearly how the proposed controller, can effectively absorb the vehicle vibration in comparisons to the PISMC method and the passive system. The body acceleration in the proposed control design system is reduced significantly, which guarantee better ride comfort.

In this paper, the peak values of the vertical acceleration are also presented. These values indicate the maximum magnitudes of the related acceleration experienced by the vehicle body or passenger. The peak values are calculated as: $\|X\|_{\infty}=\max \left(\left|x_{i}\right|\right) \quad i=1, \ldots, n$

Here, $\|\cdot\|_{\infty}$ is the $\infty$-norm. Peak values for the vertical acceleration are depicted in Fig. 5 and Fig. 6. As seen, the peak values are substantially decreased by the proposed controller. Fig. 7 shows that the wheel deflection is also smaller using the proposed controller. Therefore it is concluded that the active suspension system with the proposed control improves the 
ride comfort while retaining the road handling characteristics, as compared to the PISMC and the passive suspension system.

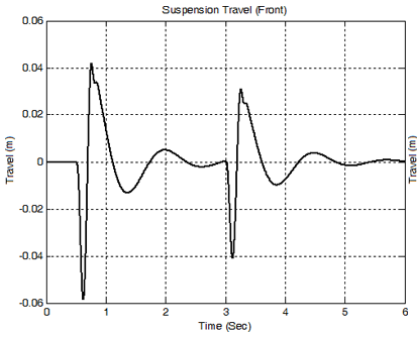

(a)

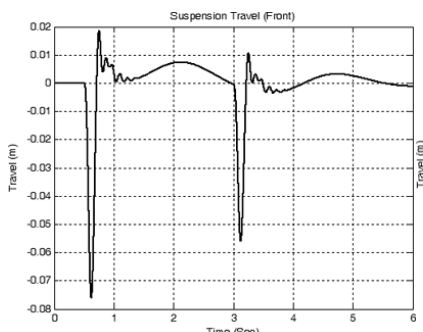

(c)

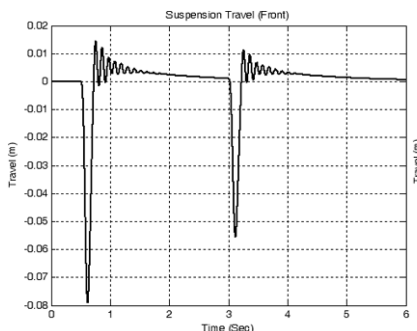

(e)

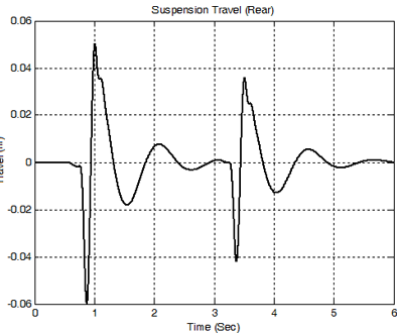

(b)

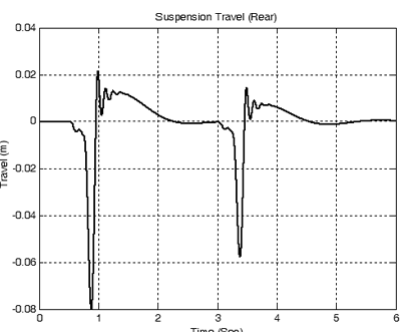

(d)

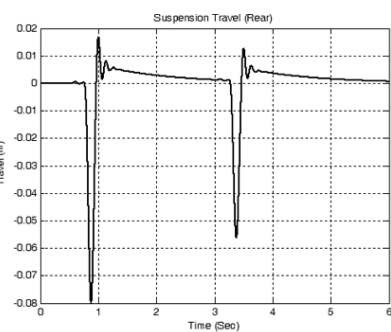

(f)
Fig. 3 The suspension travels: (a) Front passive suspension, (b) Rear passive suspension, (c) Front suspension by the PISMC, (d) Rear suspension by the PISMC, (e) Front suspension by the nonlinear optimal controller and (f) Rear suspension by the nonlinear optimal controller.

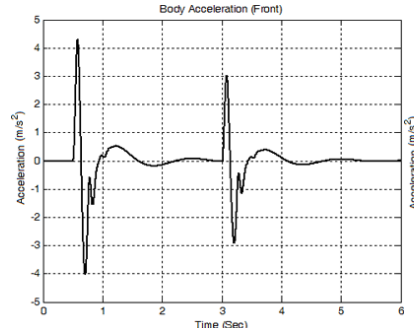

(a)

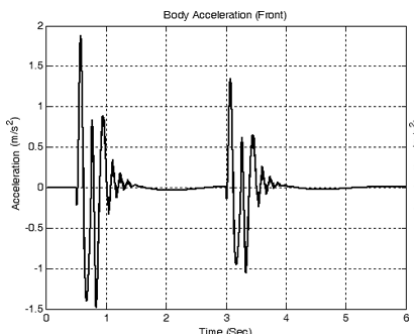

(c)

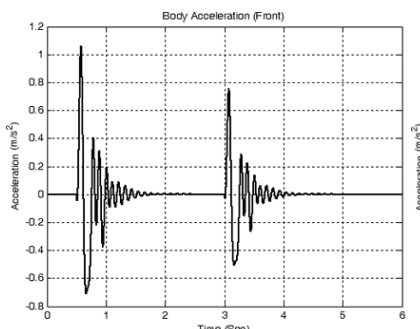

(e)

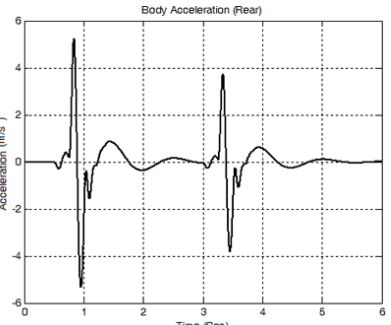

(b)

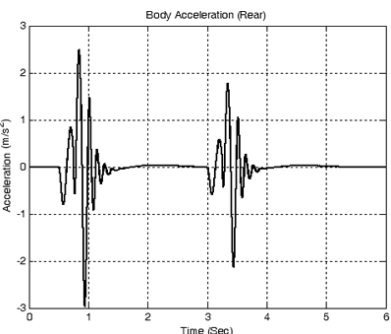

(d)

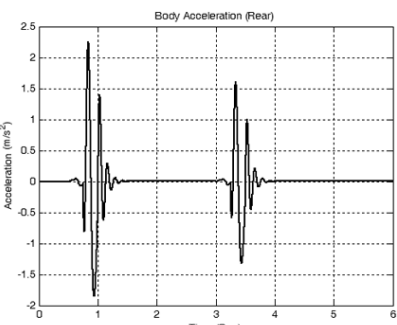

(f)
Fig. 4 The body accelerations: (a) Front passive suspension, (b) Rear passive suspension, (c) Front suspension by the PISMC, (d) Rear suspension by the PISMC, (e) Front suspension by the nonlinear optimal controller and (f) Rear suspension by the nonlinear optimal controller.

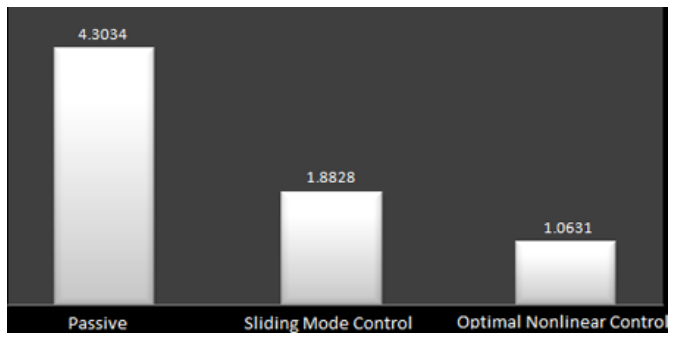

Fig. 5 The peak values for vertical accelerations of front suspension.

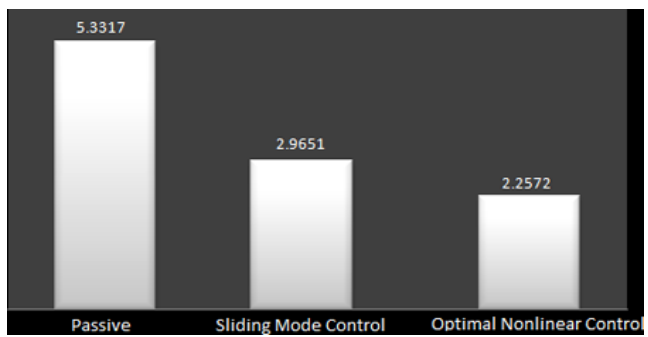

Fig. 6 The peak values for vertical accelerations of rear suspension.

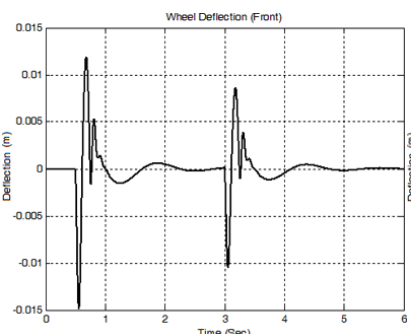

(a)

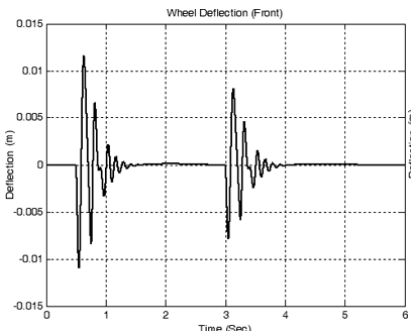

(c)

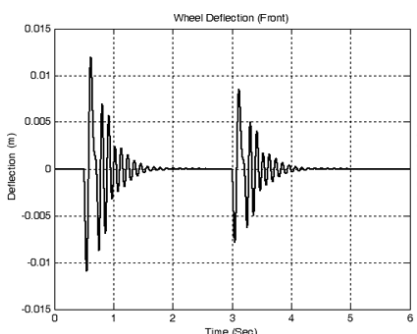

(e)

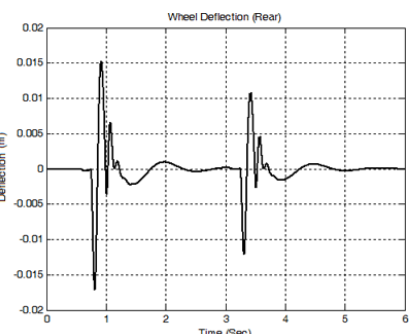

(b)

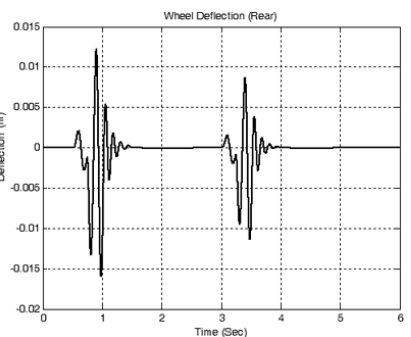

(d)

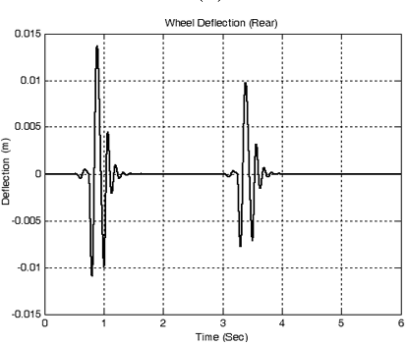

(f)
Fig. 7 The wheel deflections: (a) Front passive suspension, (b) Rear passive suspension, (c) Front suspension by the PISMC, (d) Rear suspension by the PISMC, (e) Front suspension by the nonlinear optimal controller and (f) Rear suspension by the nonlinear optimal controller.

\section{CONCLUSION}

Active suspension designs must resolve the inherent tradeoffs between ride quality, handling, suspension travel and power consumption. According to these aims, an optimization law is developed for suspension system control 
based on the states estimation and states prediction of a non-linear half car model. The proposed control law minimized the states tracking errors and led to a special case of feedback linearization. The proposed optimal non-linear control law is given in an analytical closed form that is easy to solve and online optimization is not necessary. Our proposed controller applied on sedan half car model and compared with passive suspension system and active suspension system by the PISMC method. The obtained results demonstrated that the body acceleration and wheel deflection of our proposed controller was better than passive suspension system and active suspension system by the PISMC method. Also control signals of nonlinear optimal controller were less than the PISMC method. The results demonstrated that our controller indeed proved to be effective in the inherent tradeoff between ride quality and suspension travel as compared to the PISMC method and the passive suspension system.

\section{REFERENCES}

[1] H. S. Roh and Y. Park, "Stochastic Optimal Preview Control Of An Active Vehicle Suspension," Journal of Sound and Vibration, vol. 220, no. 2, 1999, pp. 313-330.

[2] N. Yagiz, Y. Hacioglu and Y.Taskin, "Fuzzy Sliding-Mode Control of Active Suspensions," IEEE Transactions on industrial electronics, Vol. 55, no. 11, 2008, pp. 3883-3890.

[3] A. Alleyne and J. K. Hedrick, "Nonlinear adaptive control of active suspension," IEEE Transactions on Control Systems Technology, vol. 3, no.1, 1995, pp. 94-101.

[4] R. Hampo, D Hrovat, "Optimal Active Suspension Design Using Constrained Optimization," Journal of Sound and Vibration, vol. 207, no.3, 1997, pp. 351-364.

[5] N.Yagiz and I.Yuksek, "Sliding Mode Control of Active Suspensions for a Full Vehicle Model," International Journal of Vehicle Design, vol. 26, nos. 2-3, 2001, pp. 264-276.

[6] H. Okuda, Y. Tsutaka, M. Oya, O. Wang and K. Okumura, "Robust active suspension controller achieving good ride comfort," SICE Annual Conference 2007, vol. 13, 2007, pp. 17-20.

[7] S. Chantranuwathana and H. Peng, "Practical Adaptive Robust controllers for Active suspensions," Proceedings of the 2000 ASME International Congress and Exposition (IMECE), 2000.

[8] Sh. J. Wu' , H. H. Chiang, J. H. Chen, T. T. Lee, "Optimal Fuzzy Control Design for Half-Car Active Suspension Systems," Proceedings of the IEEE, vol.1, 2004, pp. 583-588.

[9] D. Hrovat, "Optimal Active Suspension Structures for Quarter Car Models," Automatica, vol. 26, no 5, 1990, pp. 845-860.

[10] H. Li, C. y. Tang, T. x. Zhang, "Controller of Vehicle Active Suspension Systems Using LQG Method," Proceedings of the IEEE International Conference on Automation and Logistics, 2008, pp. 401-404.

[11] W.H. Chen, D.J. Balanceand and P.J. Gawthrop, "Optimal Control of Non-linear Systems: A Predictive Control Approach,” Automatica, vol. 39, no 4, 2003, pp. 633-641.

[12] P. Lu, "Non-linear Predictive Controllers for Continuous Systems," J. Guidance, Control and Dynamics, vol. 17, no 3, 1994, pp. 553-560.

[13] P.J. Gawthop, H. Demircioglu and I. Siller-Alcala, "Multivariable Continuous-time Generalized Predictive Control: A State Space Approach to Linear and Non-linear Systems," Control Theory and Applications, IEE Part D, vol. 145, no 3, 1998, pp. 241-250.

[14] M. Mirzaei, G. Alizadeh, M. Eslamian and S. Azadi, "An optimal approach to non-linear control of vehicle yaw dynamics," Journal of Systems and Control Engineering, Part 1, vol. 222, no 4, 2008, pp. 217-229.

[15] M. Eslamian, M. Mirzaei and G. Alizadeh, "Enhancement of Vehicle Lateral Stability by Non-linear Optimal Control of Yaw Dynamics," Mech. \& Aerospace Eng. J. vol. 2, no 3, 2007, pp. 97-106.

[16] H.E. Merritt, "Hydraulic control systems," Wiley and Sons, 1967.

[17] M. Sanjeev Arulampalam, Simon Maskell, Neil Gordon, and Tim Clapp, "A Tutorial on Particle Filters for Online Nonlinear/Non-Gaussian Bayesian Tracking," IEEE Transactions on signal processing, vol. 50, no 2, 2002, pp. 174-188.

[18] M. Boutayeb, H. Rafaralahy, and M. Darouach, "Convergence Analysis of the Extended Kalman Filter Used as an Observer for Nonlinear
Deterministic Discrete-Time Systems," IEEE Transactions on Automatic Control, vol. 42, no 4, 1997, pp. 581-586.

[19] H. Weiss and J. B. Moore, "Improved Extended Kalman Filter Design for Passive Tracking," IEEE Transactions on Automatic Control, vol. AC-25, no 4, 1980, pp. 807-811.

[20] P. Lu, "Optimal predictive control of continuous nonlinear systems," Int. J. Control, vol. 62, no 3, 1995, pp. 633-649.

[21] D'Amato, F.J., and D.E. Viasallo, "Fuzzy Control for Active Suspensions," Mechatronics, vol. 10, no 8, 2000, pp. 897-920.

[22] J.S. Lin and I. Kanellakopoulos, "Nonlinear Design of Active Suspension,” IEEE Control System Megezine, vol. 17, no 3, 1997, pp. 45-59.

[23] U. Itkis, “Control System of Variable Structure,” Wiley, New York. 1976.

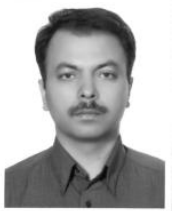

Iraj Hassanzadeh received his Ph.D. in Electrical Engineering, Control, Robotics, from University of Tabriz, Iran in conjunction with the University of Western Ontario, London, Canada and M.Sc. degrees from the University of Tabriz, in 2002 and 1994, respectively. He received his B.Sc. degree from the University of Tehran, Iran in 1991. He has been working as a Postdoctoral fellow in Mechatronics and Robotics fields at Ryerson University, Toronto, Canada for almost 2 years during 2004-2005. Since 2002, he has been with the faculty of Electrical and computer Engineering, University of Tabriz, Iran. Currently, he is director of the robotics research lab. As a team leader, he directed two robotic teams won four trophies in two international and national robotic competitions (RDC2002 and ROBOFIRE 2006). His research interests include robotics, visual servo, tele-robotics, control theory, applications and power system. He has published more than 35 international conference and journal papers in these areas. He is a member of IEEE and serves as a member of program committee of several international conferences (SPIE2005, IEEECCA2005 and IEEE Thailand2006) and several national conferences as well.

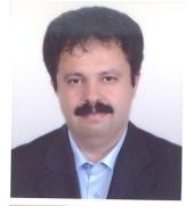

Ghasem Alizadeh received his Ph.D. in Control Engineering from Tarbiat Modares University in 1998, M.Sc. degree in Power engineering from Khajeh Nasir Toosi University of technology in 1993 and B.Sc. degree in Control engineering from Sharif University of technology in 1990. Now he is associate professor in the faculty of Electrical and computer Engineering, University of Tabriz, Iran. His research interests include Robust Control, Optimal Control, Nonlinear Control and Navigation.

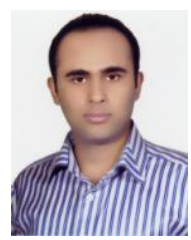

Naser Pourqorban Shirjoposht received his B.Sc. and M.Sc degrees in Electronics and control, from Azad University of Lahijan and Tabriz University in 2006 and 2009. He has just completed his M.Sc. working on Optimal Control and Nonlinear Control.

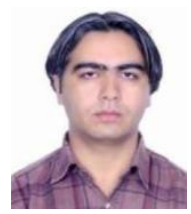

Farzad Hashemzadeh received the B.Sc. degree in Biomedical engineering from Amirkabir University of Technology, Tehran, Iran in 2003, and the M.S degree in Control engineering from University of Tehran, Iran, in 2006. Now he is Ph.D student in Control engineering in Tabriz University. His research interests include Nonlinear Pattern recognition. 